\title{
Task Design for Students' Work with Basic Theory in Analysis: the Cases of Multidimensional Differentiability and Curve Integrals
}

\author{
Katrine Frovin Gravesen ${ }^{1}$ • Niels Grønbæk ${ }^{2}$. \\ Carl Winsløw ${ }^{3}$ (D)
}

Published online: 24 August 2016

(C) Springer International Publishing Switzerland 2016

\begin{abstract}
We investigate the challenges students face in the transition from calculus courses, focusing on methods related to the analysis of real valued functions given in closed form, to more advanced courses on analysis where focus is on theoretical structure, including proof. We do so based on task design aiming for a number of generic potentials for student learning, developed from and within the theory of didactic situations: adidactic potential, linkage potential, deepening potential and research potential. The context of investigation is a first year course on analysis in which the tasks thus constructed were considered relevant to solve a number of operational problems. The experimental method involves careful a priori analysis of each task in terms of the potentials, specifically related to the knowledge at stake; this analysis in confronted with a posteriori analyses of observations of student work before and in class sessions. Two cases are analyzed in detail. While some of the potentials were partly realized, we also identified clear limitations resulting from a variety of factors, including teaching assistants' management of the class sessions and students' perception of the importance, difficulty and meaning of the tasks.
\end{abstract}

Keywords Task design $\cdot$ Real analysis $\cdot$ Didactical situations

Carl Winsløw

winslow@ind.ku.dk

1 Department of Science Education, University of Copenhagen, Øster Voldgade 3, DK 1350 København K, Denmark

2 Department of Mathematical Sciences, University of Copenhagen, Universitetsparken 5, DK 2100 København Ø, Denmark

3 Department of Science Education, University of Copenhagen, Øster Voldgade 3, DK 1350 København K, Denmark 


\section{Introduction}

In this paper, we report on work with a transition problem that is likely to occur in any university level study programme on pure mathematics, more specifically within the teaching of mathematical analysis, and usually at the undergraduate level. This problem can roughly be described as a transition from calculus courses, in which students are mainly acquiring technical knowledge that allows them to solve a wide variety of problems with functions given in closed form, to (real) analysis courses, where a main focus is on students acquiring theoretical knowledge in the sense of rigorous definitions, theorems and proofs. A more precise formulation of this specific aspect of the transition has been given by Winsløw (2008) and Winsløw et al. (2014), based on the anthropological theory of the didactic, and it constitutes the first major focus of the paper. Another important aspect of the transition comes from the ambition (particularly in research intensive universities) that students should progressively engage in "research like activities". By this, we mean mathematical activities which approach those of mathematics researchers (cf. Madsen and Winsløw 2009). This aspect of the transition is the second main focus in the paper.

More concretely, we present

- A methodological approach to designing exercises for students that could facilitate the transition;

- A theoretical analysis of two selected cases, which illustrate different theoretical and observed "potentials" (defined in the next section)

- An analysis of the outcome of students' work with these two exercises (in written forms as well as during class time), focusing on the topics of differentiability and integrability of functions of several variables.

As exercises can be considered "cores" of situations of learning, we have developed a theoretical framework of design and analysis of such situations which is sensitive to the transitional phenomena outlined above. This framework is a main point of the paper. We should also stress that the cases presented here were not selected to showcase unconditional successes (in fact, we will observe several shortcomings); instead they illustrate how the framework permits both a control of the design process (focusing on very specific didactic potentials), and the corresponding critical analysis of observations.

We now proceed to present this framework in detail.

\section{Theoretical Framework and Research Questions}

The main theoretical basis of this paper comes from the Theory of Didactical Situations (Brousseau 1997), shortened to TDS in the sequel. An overview of earlier adaptations of TDS to the university context is given by González-Martín et al. (2014). We will in particular consider the following types of situation related to different settings of work on a given exercise, as discussed in more detail by Joubert (2013): 
- The situation of devolution is the students' encounter with the formulation of the exercise, together with any further instructions given on what students should do with it - unlike devolution in a classroom situation, teachers and students do not have the possibility to interact further before the students accept (or not) to engage with the problem;

- Adidactic situations of action and formulation occur whenever students (individually or in groups) work with solving the exercise - at home, or in a classroom where the teacher does not instruct the students on the solution;

- Adidactic situations of validation occur when students verify (or attempt to verify) the quality of their solution, without being instructed about this by the teacher or the exercise text;

- Didactic situations of action, formulation and validation occur when student solutions are presented and verified in an interaction between students and teachers;

- Situations of institutionalization occur when exercise solutions are endorsed by the institution, as correct, for instance when the teacher presents a solution in a classroom setting or in writing ("standard solutions"), usually in continuation of one or more of the previous situation.

These rough definitions should be understood in the light of the more abstract notion of Situation, which is an ideal or observed "system of relationships between students, a teacher, and a milieu (...) [where the milieu is] the set of material objects, knowledge available, and interactions with others, if any, that the learner has in the course of said activity" (González-Martín et al. 2014). In general, the milieu evolves in time and may be modified by students and teachers alike. The problem is handed over to the students in the situation of devolution, and it is a central focus point for the students' interaction with the milieu in all of the above situations. In the situations we study, the problem is stated formally as an exercise.

A central and difficult notion of TDS is that of adidacticity. In classic situations of TDS it refers to phases of classroom work where the teacher does not instruct, but where the students are supposed to learn by adapting their knowledge to a milieu, devolved and regulated by the teacher. In the adaptation outlined above, adidactic situations include also student work with exercises at times when they cannot interact directly with the teacher and where, in particular, the teacher cannot modify the milieu; to compensate for this, exercises are sometimes supplied with "hints" the students are assumed to need, or divided into small and supposedly manageable parts. The adidactic situations related to exercises are usually considered important parts of students' work and learning in a university course. It is therefore crucial to maximize the adidactic potential (Hersant and Perrin-Glorian 2005) of the situation, i.e. the potential for students to develop new knowledge on their own as they work with the exercise, drawing on other features of the milieu like established knowledge and shared experience, interaction with peers, and resources which are assumed to be available, such as course textbooks, Internet etc. As this possibility depends crucially on the exercise itself, we shall refer to it as the adidactic potential of the exercise.

On the other hand, the organization of didactic situations of formulation and validation is in part based on an assumption that not all students will be able to complete formulation and validation of solutions by themselves - or, at least, that a 
shared work on formulation and validation is needed before the crucial situation of institutionalization. Institutionalization refers here to the establishment of knowledge developed in the situation as not only valid within the group, but also as consistent with the aims of the course and, more generally, of the institution in which the situation takes place. The knowledge then becomes available as a resource for future situations along with other parts of established knowledge as found, for instance, in textbooks.

Three more specialized features of the exercises to be designed will also be pursued in this paper:

- Linkage potential, i.e. the potential of the exercise to establish links between students' "old knowledge" (mainly from calculus) and new knowledge, which is closely related to the transition problem outlined in the introduction; we notice that this is also crucial to the adidactic potential, to the extent it enables students to invest old knowledge rather than knowledge in development (Hersant and PerrinGlorian 2005). In many cases (and in both cases studied in the sequel) this is realized more specifically by including familiar objects from calculus in milieus for new, more abstract problems from analysis.

- Research potential, i.e. the potential of the exercise to let students engage in activities which are similar in kind to the scholarly activity of research mathematicians, as investigated empirically by several authors such as Burton (2004), Misfeldt (2006), Madsen and Winsløw (2009) and Ouvrier-Buffet (2011). We aimed to design exercises that could engage students in the following ten types of research-like activity (inspired by the previous references as well as our own experience with mathematical research):

A1. Investigate given special cases, in view of answering an abstract or general question

A2. Construct or identify special cases that would be relevant to A1

A3. Read and reconstruct a proof or part of it, in view of answering some question

A4. Reformulate a theorem, e.g. for a special case or condition

A5. Formulate new proof by "mimicking" a known one (e.g. for an analogous case; related to A3)

A6. Employ a non-formal "heuristic" representation of a mathematical object, to investigate it

A7. Formulate hypotheses for a given question

A8. Search for, and evaluate, relevant knowledge for an inquiry (among colleagues, online, ...)

A9. Develop and formalize relations between two or more results.

A10. Produce or validate definitions, usually in connection with one of the other activity types.

The above is our current research hypothesis on what activities could be most relevant to strive for in a first course on analysis; we can only illustrate some of them in this paper. In the conclusion, we return briefly to broader aspects of our experience with their use. 
- Deepening potential, i.e. the potential of the exercise to let students elaborate their knowledge of notions (mainly established by formal definitions), results (mostly theorems) and methods (as found in proofs) which are central to the learning goals of the course ("knowledge in progress", in the sense of Hersant and Perrin-Glorian (2005)). For instance this may occur as students construct new inferences between results or definitions, or as they reformulate, generalize, or instantiate them. We note that the research literature holds less relevance to this potential, which is nevertheless crucial for our context (cf. section "Context and Methods").

Each of the four potentials of an exercise has, in principle, two kinds of value: theoretical and observed values, which are to be analyzed, respectively: a priori (from the formulation of the exercise itself, and what is known about the context), and $a$ posteriori (from observations of actual student work).

Our research questions can now be formulated as follows:

RQ1. How do the potentials described above function as design guidelines to produce exercises that could improve students' experience and learning in the context of a first course on analysis, focusing on the foundations of calculus in one and several variables?

RQ2. What realizations of the four potentials are observed in practice?

RQ3. What main explanations can be given for any differences among theoretical and observed values?

We notice here that RQ1 can only be answered with respect to methods actually imagined and carried out, so the answer will be highly partial in the sense that it relies on the strategy adopted in our study and described below ("Context and Methods"); while the main discussion of designs will be done for two specific cases (exercises), we also discuss broader observations of the outcomes of the design process and its effects ("Perspectives"). Similar methodical constraints apply to RQ2 and RQ3, but our empirical data will provide more direct, even if partial answers to these questions.

\section{Context and Methods}

The concrete context which led us to develop the generic aims for exercise design laid out in the previous sections is a course, Analysis 0, given in the second semester of the bachelor programme in (pure) mathematics at the University of Copenhagen. During their first semester, the students have courses on calculus (one and several variables, vector analysis, differential equations) and linear algebra. These courses are also taken by students of a number of other science programmes and the main focus is on technical skills with concrete functions, matrices and so on, and very little on mathematical theory. The contents of Analysis 0 can be considered a review of the topics from the calculus part of the first semester, but now treated from a much more theoretical angle which also necessitates an indepth work with the topology of $\mathbb{R}^{n}$ (neighborhoods, open and closed sets, completeness, limits and continuity, etc.).

The more theoretical focus also appears in the final evaluation of students' learning: while the first semester courses are assessed based on written solutions to standard exercises, the 
examination in Analysis 0 is oral. The examination is based on a list of 12 "questions" known in advance. Each question consists of a few keywords (e.g. "The Riemann integral" or "Green's theorem") which, in essence, require the students to state and prove one or more theorems from the textbook. At the beginning of the examination, the individual students draw one question from the list; the students then have 30 min preparation time, followed by 25 min examination where they present the proof(s), often in interaction with the examiner. The grade is given based on this performance alone. A condition for examination admittance is that one hands in three sets of written exercises during the course, but there is no effective control that all of these solutions are individually produced.

The course comprises $4 \mathrm{~h}$ of lecture and $5 \mathrm{~h}$ of class teaching per week for 7 weeks. The lectures cover the selected parts of the syllabus, extracted from the textbook (Eilers et al. 2015). The class sessions focus on presentation of exercises and on rehearsal by volunteering students of the "questions" for the oral exam. The lectures are given by a senior faculty member, while the class sessions are run by graduate students of mathematics. The course has about 300 students, organized in 10 classes for the work with exercises.

A recognized weakness of the course in later years - consistently reported by teaching assistants - is that a few weeks into the course, almost all students come to the class sessions without having done or even attempted to do the exercises to be treated. The main explanation given is the difficulty of the exercises, which are mainly taken from the textbook. Another factor is, presumably, that students focus on preparing for the oral exam, and do not see how the solution of somewhat advanced tasks can support that preparation. Moreover, students are reported to find the course as a whole "much more difficult" and even "a big jump" in relation to the first semester courses; time constraints is a major factor mentioned by students in their evaluations of the course. Thus, there is both a problem of alignment of examination and exercise sessions, and of adapting the exercises to the capacity of the students. As regards the last problem, one could, of course, imagine that time would be allotted within the class sessions to provide guidance to the students' personal work, should they not have been able to solve the exercises before; but at present, this has not been considered feasible within the course time allotted. Other constraints include an impossibility to change the examination format significantly, or to make other drastic changes in the course beyond modifying the exercise inventory. These impossibilities are related to more global constraints in the study programme and within the institution and will not be examined more closely in the present paper.

The outcome is that students need exercises which are aligned with the examination and which they can conceivably solve by themselves, at least in part. This motivates the construction of new exercises with higher adidactic potential and linking potential, and with a deepening potential explicitly related to the theorems and proofs implied in the examination questions. The additional strive for research potential is related to a development project of the university, aiming to give undergraduate students authentic experiences of research or research-like work in their field, an ambition which is also evident in the overall directions of the bachelor programme in mathematics.

\section{Exercise Design Methodology}

As noted by Joubert (2013), exercise design should be based on an explicit epistemological analysis of learning potential. At the same time, our design was supposed to 
address the broader problem of transition from calculus courses to a first course in analysis. Thus, our design could not only be guided by a priori investigation of specific target knowledge and the conditions under which a given milieu could suffice for students to attain it. The guidelines should also relate to more generic features of learning potentials that would go beyond the individual exercise. The four potentials, introduced above, emerged early in our initial discussions of the course. They were initially formulated more informally but the theoretical framework proved useful to isolate the various shortcomings of students' previous experience with exercises in the course. Then, it was important to realize that all exercises needed to have adidactic potential to be both worthwhile and feasible, and our design methodology explicitly addresses this point (we return later to needs for redesign with this in mind). It was equally important to decide that the other three potentials were not necessary in all exercises.

Our design methodology is naturally shaped by the constraints of the context - in particular the need to align the exercises with the examination by linking each of them explicitly to one of the 12 questions.

This led to the following design principles which concretize the way the four potentials were pursued:

- The adidactic potential was considered in the light of the earlier experiences mentioned above (too "difficult" exercises leading to passive students), and it was a high priority to ensure that all exercises offered appropriate milieus for the tasks proposed, so that students could find at least initial or partial answers prior to the class sessions;

- The deepening potential was pursued through formulating exercises that would lead students to reflect on specific details and overall structure of proofs to be presented at the oral exam;

- The linking potential would be achieved if the milieu proposed by exercises enables students to relate a theorem, definition or proof from the course to specific mathematics from the first semester calculus course or high school analysis;

- The research potential was mainly identified with "open questions" (i.e. with no given answer - as opposed to most classical exercises, beginning "Prove that..."). They were designed purposefully to lead students into one or more of the activity types A1-A10.

At a more practical level, a structured procedure for the design of exercises was set up in order to invest the different competences available in the team, consisting of: course leader Søren Eilers (analysis researcher), Anna Ebbesen (experienced teaching assistant in An0), Carl Winsløw and Niels Grønbæk (researchers in didactics of mathematics and analysis), and Katrine Gravesen (master student in mathematics, specializing in didactics of mathematics). The procedure was set up as follows:

Step 1. first versions of exercises were produced by Anna Ebbesen and Niels Grønbæk, based on a reading of the corresponding sections of the textbook and the above design principles;

Step 2. the first versions of the exercises were solved by Katrine Gravesen, to gauge the adidactic potential with a student who had the course some years ago; 
Step 3. the exercises were then revised by Katrine Gravesen and Carl Winsløw in collaboration, focusing specifically on achieving the four design principles explained above; at the same time, additional exercises were formulated to complement the set.

Step 4. finally, the exercises were reviewed by Søren Eilers, who also made final changes and adaptations, and shared the result with the rest of the team (this part of the procedure focused on aligning the exercises with other decisions made on the course).

This "four step production line" ran for 10 weeks (with about two new sets being launched each week and each step lasting about 1 week). The result was a collection of 57 exercises, which we refer to as "R-exercises" to distinguish them from other exercises treated in the class sessions; the $\mathrm{R}$ indicates the ambition of inducing research like situations, as a central but not exclusive aim of the design.

\section{Research Methodology}

The a priori analysis (Hersant and Perrin-Glorian 2005) of the R-exercises began during the above design process, especially in step 1 and 3 . At this point, the adidactic potential was evaluated mainly in terms of (1) the experienced difficulty of the exercise (step 2 of the design process) and (2) the potential of the exercise to make a milieu available, partially based on knowledge from the previous courses, which would be sufficient to produce a complete solution. All four potentials were explicitly assessed in step 3, in the concrete terms of the design principles.

More complete a priori analyses have then been carried out with a smaller selection of the final R-exercises (i.e. after step 4 of the design process). Such an analysis is always done in terms of an explicit presentation of the wider mathematical context of the exercise, and involves a close examination of the specific target knowledge and milieu offered by the exercise, in relation to students' prerequisite knowledge; the goal is to obtain a finer analysis of the potentials that could be realized and the different obstacles which could be foreseen (Brousseau 1997). These analyses are presented in the first authors' master thesis under the supervision of the last author (Gravesen 2015), which also contains detailed analyses of data from the students' work with the exercises (as explained below). In this paper, we present a synthesis of the a priori and a posteriori analyses of two R-exercises in this paper; these two were selected in order to present both obstacles and realizations for each of the four potentials.

The a posteriori analyses of the didactic situations induced by the R-exercises form the core of the master thesis of Gravesen (2015); the present paper draws substantially on that work. In particular we make use of the data collected in view of answering RQ2 on students' work with the exercises:

- collection of written homework by seven focus group students, done on the R-exercises prior to the class sessions

- video recordings of all class sessions in one specific class, focusing on capturing the blackboard development of solutions and the corresponding voices of students and teaching assistant; 
The video recordings were transcribed and divided into realized situations (treatments of exercise questions) and episodes (corresponding to shifts in the distribution of responsibility among teaching assistant and students, i.e. to different didactic contracts), as explained by Hersant and Perrin-Glorian (2005). Confronting the students' contributions (in homework and during the class situation) with the a priori analysis, we discuss the ways and extent to which the targeted potentials were realized.

The teaching assistant did all of the blackboard writing during almost all exercise sessions, while inviting the students to contribute orally at central points. This means that the student productions made prior to the sessions constitute the most important material to gauge what students could actually achieve by themselves. When asked, the teaching assistant invoked time pressure as a reason for this way to conduct the sessions. While that reason is questionable, a change to this pattern would require explicit interventions. In fact, all teaching assistants observed used a similar approach.

As a complement to these primary sources of information on students' work, various other data sources were available, but will only marginally be drawn on here. The first author conducted two interviews with the focus group students, to validate and clarify observations made from the above. She also observed a large number of oral examinations, in order to examine implicit or explicit effects of the R-exercises in this setting. Finally, we had access to all data from students' evaluation of the course, and to the results from the final evaluation (oral exam) of the students.

The two cases (exercises) analysed in this paper were selected carefully to present a certain variety of potentials and obstacles identified in the study, in order to enable a substantiated discussion of the more generic research questions formulated above. In particular, they illustrate a variety of the activity types which were used to realize the research potential. The two cases also illustrate two typical and different needs for redesign of the exercises concerned (Case1 : enrichment of the milieu for technical questions; Case 2: alter devolution of abstract problems, even when a calculus milieu for them has been established).

\section{Case 1: Differentiability in $\mathbb{R}^{k}$}

The students worked with the following exercise during the fifth week of the course; the theorem mentioned in question $b$ ) is cited below.

a) Decide whether the following functions $f: \mathbb{R}^{2} \rightarrow \mathbb{R}$ are differentiable:

$$
\begin{gathered}
f(x, y)= \begin{cases}\frac{x y}{x^{2}+y^{2}}, & \text { if }(x, y) \neq(0,0) \\
0, & \text { if }(x, y)=(0,0)\end{cases} \\
f(x, y)=(x y)^{\frac{1}{3}} \\
f(x)= \begin{cases}\left(x^{2}+y^{2}\right) \cdot \sin \left(\frac{1}{\sqrt{x^{2}+y^{2}}}\right), & \text { if }(x, y) \neq(0,0) \\
0 & \text { if }(x, y)=(0,0)\end{cases} \\
f(x, y)=\sqrt{x^{2}+y^{2}}
\end{gathered}
$$


b) Use your results to comment on the assumptions in Theorem 6.11 and its result.

c) Make your own "recipe" to decide whether a given function of two variables is differentiable.

It is to be understood here that "differentiable" means throughout $\mathbb{R}^{2}$, in particular at $(0,0)$.

\section{Mathematical Context}

Students will naturally refer to sec. 6.2 of (Eilers et al. 2015), entitled "Differentiability in several variables". The most relevant elements here are (everything concerns functions $f: \mathbb{R}^{k} \rightarrow \mathbb{R}$, defined on some open $\Omega \subseteq \mathbb{R}^{k}$, and the below are outlines, rather than exact translations from the textbook):

- Definition 6.5: $f$ is said to be differentiable at $a \in \Omega$ if there is $c \in \mathbb{R}^{k}$ so that $\frac{f(\boldsymbol{x})-f(\boldsymbol{a})-\boldsymbol{c} \cdot(\boldsymbol{x}-\boldsymbol{a})}{\|\boldsymbol{x}-\boldsymbol{a}\|} \rightarrow 0$ as $\boldsymbol{x} \rightarrow \boldsymbol{a}$; in this case $\boldsymbol{c}$ is called the derivative vector of $f$ at $\boldsymbol{a}$.

- Theorem 6.6: If $f$ is differentiable at $\boldsymbol{a} \in \Omega$ then $f$ is continuous at $\boldsymbol{a}$.

- Theorem 6.7: If $f$ is differentiable at $\boldsymbol{a} \in \Omega$ with derivative vector $\boldsymbol{c}$, then $f$ has directional derivatives $D_{v} f(\boldsymbol{a})$ at $\boldsymbol{a}$, in all directions $\boldsymbol{v}$. In fact $D_{\boldsymbol{v}} f(\boldsymbol{a})=\boldsymbol{c} \cdot \boldsymbol{v}$.

- Theorem 6.11: If $f$ has partial derivatives $D_{1} f, \ldots, D_{k} f$ in every point of $\Omega$, and if these are all continuous at $\boldsymbol{a}$, then $f$ is differentiable at $\boldsymbol{a}$.

We notice here that one of the oral examination questions is Differentiability in $\mathbb{R}^{k}$, with Theorem 6.11 being the recommended part to prove; but of course, students drawing that question should ideally know all of the above in a coherent way. In the book, several examples are given to illustrate the theorems individually and in particular that the converses to Theorem 6.7 and 6.11 are not true. We notice here that while the Definition and Theorem 6.6 resemble "old knowledge" for the case $k=1$, Theorems 6.7 and 6.11 do not have counterparts in that case and must be considered entirely "new knowledge". At the same time, these theorems provide crucial links between old and new knowledge, if one realizes that they provide a necessary resp. sufficient condition for differentiability in several variables, and that these conditions concern differentiability of certain one variable functions (besides continuity in several variables, in the case of 6.11). The textbook does not state explicitly how the different elements above may serve to prove or disprove differentiability.

\section{A Priori Analysis of the Situation}

The target knowledge of the situation is, in a narrow sense, practical techniques to determine whether a function in several variables is differentiable, and explicit knowledge of necessary and sufficient criteria that can be extracted from the relevant elements from the textbook. In a broader sense, students should get a better situated knowledge of differentiability of functions of several variables by overcoming certain obstacles in concrete situations: first, the didactic obstacle (established in high school) that "differentiability of $f$ " means that "you can differentiate $f$ " in the sense that some algorithm applies to do this in all cases encountered; and the epistemological obstacle that since $\left(D_{1} f(\boldsymbol{a}), \ldots, D_{n} f(\boldsymbol{a})\right)$ is in fact the derivative of $f$ at $\boldsymbol{a}$ when it exists, differentiability means 
that the partial derivatives can be computed. A similar didactic obstacle, related to Theorem 6.11, is the belief that differentiability simply means existence and continuity of the partial derivatives.

The students can be expected to be familiar with ordinary differentiation and computation of partial derivatives; they can be trusted to affirm the differentiability of functions in closed form and to know the simplest exceptions, such as $\sqrt{x^{2}}$ at 0 . However, they may not realize immediately what the four elements from the textbook can be used for. In particular, to solve the exercise, one needs to realize that only the definition gives (as it should) necessary and sufficient conditions, but they are often hard to apply directly; Theorem 6.11 gives sufficient conditions, while the remaining two theorems give necessary conditions. Part a) provides four concrete episodes of actions which allow the students to work with these criteria, while part b) and c) require formulations at a more abstract level.

In outline, one can handle question a) as follows (the details are left to the reader, while some appear in the a posteriori analysis): (1) Non-differentiable (by 6.6), (2) Non-differentiable (by 6.7), (3) Differentiable (by 6.5), (4) Non-differentiable (by 6.7). For question $b$ ), the students should use example 3 . from a) to show that the conditions given in that theorem are not necessary for differentiability and conclude that the converse result is false; this is not shown from examples in the textbook and is thus new knowledge even in relation to the course. As for c), the word "recipe" should lead to some order in which to do the investigation of a function, such as plotting it on the computer to see if the graph looks smooth or not, and then some instructions on the use of the two relevant elements in either case, based on the experiences from a).

We notice in passing that Theorem 6.11 does not apply to any of the examples in a), except of course at the trivial points away from $(0,0)$. This largely negative point may surprise the students, given that the textbook introduces this theorem as "salutary" when it comes to decide on differentiability.

The exercise has adidactic potential as students should be able to do some first relevant steps in a), such as computing partial derivatives or doing a plot of the functions; these may help towards forming a hypothesis and then finding a relevant strategy to produce a formal argument. To solve a) completely requires formal reasoning about unfamiliar, given functions, while drawing on knowledge (at best) in progress for the students. It is evident that successful completion of a) provides useful input to b) and c), but less with b), that would seem to require that the students have tried very hard to use Theorem 6.11 in a). The textbook's emphasis of that theorem could, however, make this plausible.

The linkage potential is evident from the need in a) to reinvest computational skills from first semester calculus, to find concrete partial derivatives from algorithms as well as from the definition. The deepening potential concerns mainly definition 6.5 and the meaning of the three theorems cited above; at least the students should become more familiar with the definition and the fact that one direction implications in each of the three theorems. We note that the proof of Theorem 6.11 is normally considered the main focus of the oral examination question, and the proof is only marginally supported by the exercise; however, Definition 6.5 is central to that proof (together with the ordinary mean value theorem which is not touched upon here). The exercise finally has research potential to engage the students in activities of type A6 and A7 in a), A1 and A7 in b), and A9 in c). 


\section{A Posteriori Analysis of the Situation}

Five of the seven focus group students had done some written homework related to this exercise prior to the session where it was on the agenda, but none of them have done all three questions. In fact, four of the five students mainly get to compute partial derivatives of the first two or three functions in a), and even for the first function, only two of them get to the correct answer (non-continuity and hence non-differentiability by Theorem 6.6 - only one of the two states the theorem explicitly to support the conclusion). Two unproductive strategies otherwise dominate the work accomplished: vain attempts to use Theorem 6.11, and attempts to compare mixed second order derivatives (this was covered in the preceding week, but is evidently irrelevant for the present problem). This confirms at least two elements of the a priori analysis: the adidactic potential resulting from the familiarity of the students with partial derivatives as a way to "differentiate" functions of two variables, given in closed form; and the didactic obstacle of the perceived strength of Theorem 6.11, which in fact is important in many cases, but cannot be used for the cases in a). Only one of the students gets as far as question c); not surprisingly, this student is also the one who did most of a) correctly. This student proposes the following "recipe" (translated from Danish):

If you can show that all partial derivatives are continuous, you are done, but the theorem does not say anything about the case when the partial derivatives are not continuous. If you want to show that a function is not differentiable, it can be useful to look at the directional derivatives, and if one of them does not exist, it [the function, authors] is not differentiable. If one can show from the beginning that the function is not continuous, then it [the function, authors] cannot be differentiable.

It is remarkable that even this student puts the methods based on Theorem 6.11 first, even if it has not worked for any of the four functions despite eager attempts to use it. Otherwise, the methods which this student actually succeeded to use are mentioned in a somewhat unmotivated order.

Based on these observations, it appears likely that few (if any) students have solved the whole exercise beforehand, while those who have tried have at least computed some partial derivatives in attempts to use Theorem 6.11. The observed adidactic potential of the exercise thus consists mainly in the availability of some initial strategies, while the main parts of the new knowledge were not reached by the students prior to the didactic situation in class. Regarding research potential, the computation of partial derivatives are the only (mostly unsuccessful) activities of type A7 which the students engage in for a); thus, none did a plot of the four functions (away from the origin) as a way to form a hypothesis.

In class, a total of $50 \mathrm{~min}$ is used on this exercise; the teaching assistant does, as usual, all board writing, while involving individual students through questions which become successively less open as the presentation progresses. The teaching assistant is clearly aware of the time constraints and the total list of exercises to be covered in the session.

For each of the four functions in a), the teaching assistant (TA) initially asks the students for ideas, which are more or less in the direction of using Theorem 6.11; 
certainly they all involve computing partial derivatives, occasionally at $(0,0)$. She develops the computation far enough to suggest that the strategy is not productive, in particular (in (3)) that although the partial derivatives are not continuous at $(0,0)$, nothing can be inferred from that. It turns out to be more difficult for her to elicit student generated hypotheses that actually work. We sometimes observe outright Topaze effects (Brousseau 1997), as in the discussion of the function in (1) (following a lengthy discussion of partial derivatives):

TA: Let's go back and look at our function [points to the expression of $\mathrm{f}$ in a), (1)]. What is needed for this one to be differentiable? The easiest criterion for something to be differentiable?

Student 1: The $C^{1}$-criterion or what?

TA: Yes almost, or you have got the right part. Can something be differentiable if it is not continuous?

Student 2: No.

Nothing in the milieu - and certainly not the defining expression of $f$ which is being pointed at - suggests the approach which the teaching assistant tries to get a student to suggest. So, she gives it away, and thus reduces the task dramatically to that of showing non-continuity of $f$ at $(0,0)$. However, she still tries to include the students in generating ideas for solving this task. Pointing again to the expression $\frac{x y}{x^{2}+y^{2}}$ of $f$ away from the origin, a collective phase of questions and answers lead to the insight that approaching the origin along the axis does not help to show non-continuity. Then she continues:

TA: So that's the big trouble. How do I find where it goes wrong?

Student 1: If you let $x=y$, you get in trouble.

TA: It shall be no secret that that's my choice, but how do I find out that that's my choice?

The teaching assistant is not satisfied with having a"good" choice of direction from one student, and as no student answers the last question, the teaching assistant mentions the possibility of looking at the graph of $f$. But no graph is drawn or shown, so neither the exercise nor the intervention by the teacher succeeds in creating a milieu with adidactic potential related to the use of graphs, which could also be crucial to realize the research potential linked to the heuristic investigation (A6). Instead, the teaching assistant proceeds to the algebraic verification that $\lim x \rightarrow 0 f(x, x) \neq f(0,0)$.

The remaining three cases in a) are treated similarly with an acceleration of time that reflects the teaching assistant's management of time: at first, she takes time for more student contributions and even errors; then she accelerates, as the contract seems to 
dictate that all questions be answered on the blackboard (at least summarily). Following this pace, the answer to $b$ ) is institutionalized by the teacher, pointing out that although Theorem 6.11 is important, it does not give a necessary condition for differentiability, and in fact was not useful in a).

Given this relative dominance of the teaching assistant in most of the treatment of a) and b), it is remarkable that several of the potentials appear again in the students' contributions to c): the teaching assistant assumes the posture of a secretary who notes the contributions of the students to the "to-do-list" for deciding if a given function is differentiable (while, as we shall see, she does more):

TA: ...so now let's at least write up some things which can make your life easier. What's the first we do?

Student 1: Check it's continuous.

TA: Check it's continuous. I think that this is a really good idea. And what's our favorite way to check if something is continuous?

Student 2: Check $(0,0)$ and see what it does there.

TA: Check all directions towards, I call it, a bad point. Most often $(0,0)$ but not always. (...) Now I just write, 'cause I insist and hope some of you will do it, [writes:] Draw! It's a really really good idea. Just to get an idea where you are going (...) and ifyou use one of these, so to say, "trick ways", so say, boom, it's not differentiable.

Here, student 2 does not manage to abstract from the four examples in a), and the teaching assistant validates ("not always") and adds precision ("check all directions"). She then returns to the idea of using the graph, alluded to above. We note here that no graph is ever shown in the situation, so the usefulness of the idea remains a claim. By "trick ways", the teaching assistant may also allude to the strategy based on Theorem 6.7, which she returns to later. As the dialogue continues, the strategy based on Theorem 6.11 is also explained and clarified. Then "the absolutely most cumbersome method" (to quote a student) is formulated, namely that based directly on Definition 6.5:

TA: So we have found the partial derivatives at these places where we can't find out [about continuity of the partial derivatives, authors].

Student 3: Then you use your definition from before and look at the difference between, that is you guess your vector and look at the difference between $\Delta f$ and that vector times $\Delta x$.

TA [writing]: "Guess a c and use the definition".

The whole episode focused on c) lasts about $7 \mathrm{~min}$ and consists in individual and collective production (Hersant and Perrin-Glorian 2005) that involves a total of 8 
students, with the teaching assistant validating (more or less explicitly) and at the same time institutionalizing the "list" as she rephrases the students' suggestions on the blackboard. A full analysis of the episode would lead us too far here and involve also the identification of didactic obstacles which were not visible in other parts of the situation, such as a mistaken idea that the computation of second order partial derivatives could somehow help to decide on differentiability.

The main point here is that both the research potential (A9) and the deepening potential are realized in this episode, even if large parts of the preceding episodes on a) and b) had limited student contributions. The focus group students who did most of this exercise (including c) before class, as mentioned earlier, were not present at the class session. However, even students who had done no written preparation for this exercise contributed with essentially correct general strategies for c), based on the work presented for the four examples in a). These observations contribute to the interest of situations of collective production like the above generated by question c), especially for students who do not succeed or engage with the tasks before the didactic situations in class.

\section{Case 2: Curve Integrals}

The second case concerns the following exercise, worked in the seventh course week (an explanation of the context, which is necessary to understand the enunciation, follows):

a) According to the textbook, one can convince oneself of the uniqueness of the curve integral in Definition 7.20. Use Definition 7.20 to show that there does not exist two different $I_{1}$ and $I_{2}$ with the desired property.

b) Compare with R-exercise 10.3.

c) Read the comment after Definition 7.20 and explain why the curve integral is a geometric object.

Here, R-exercise 10.3 asks the students to show that the expression $\int_{a}^{b} \mathbf{V}(\mathbf{r}(t)) \cdot \mathbf{r}^{\prime}(t) d t$ is invariant under smooth, direction preserving reparametrization of $\mathbf{r}$, then explain that the curve integral is independent of the choice of a $C^{1}$-parametrization; the last part would invoke Theorem 7.22 below.

\section{Mathematical Context}

The above R-exercise is clearly linked to the examination question "The curve integral" and hence to section 7.4 in (Eilers et al. 2015), with the same title. It concerns essentially the basic definition (p. 230). The detailed formulation is sufficiently important for the sequel that we give a full translation, while we do assume that the notation and terminology is familiar to the reader: 
Definition 7.20. Let $\Omega \subseteq \mathbb{R}^{k}$ be given. Let $\mathbf{V}: \Omega \rightarrow \mathbb{R}^{k}$ be a vector field in $\Omega$, and let $\gamma$ be a continuous curve in $\Omega$, with parametrization $\mathbf{r}:[a, b] \rightarrow \mathbb{R}^{k}$. We say that the curve integral of $\mathbf{V}$ along $\gamma$ exists, if there is a number $I \in \mathbb{R}$ with the following property: for all $\varepsilon>0$ there is $a \delta>0$ such that for every partition $D$ of $[a, b]$ with fineness $<\delta$ and for every choice of intermediate points, the corresponding middle sum satisfies $\left|I-\sum_{i=1}^{n} \mathbf{V}\left(\mathbf{r}\left(\tau_{i}\right)\right) \cdot \mathbf{r}_{i}\right|<\varepsilon$. In this case, I is called the curve integral of $\mathbf{V}$ along $\gamma$, and is written $\int \mathbf{V} \cdot d \mathbf{r}$.

The remark following this definition, alluded to in b) and c) of the above exercise, reads as follows:

One can easily convince oneself that there can at most be one number I with the desired property. It is also easy to see that the curve integral is a geometric object, in the sense that it does not depend on the parametrization of $\gamma$.

The section includes otherwise two main results. First, an existence theorem which is not relevant here. Then, Theorem 7.22 which states (under the condition that $\boldsymbol{V}$ is continuous and $\mathbf{r}:[a, b] \rightarrow \mathbb{R}^{k}$ is a $C^{1}$-parametrization of $\gamma$ ) the useful formula $\int_{\gamma} \mathbf{V} \cdot d \mathbf{r}$ $=\int_{a}^{b} \mathbf{V}(\mathbf{r}(t)) \cdot \mathbf{r}^{\prime}(t) d t$, reducing the computation of curve integrals to "an ordinary Riemann integral" (p. 232). The proof of this theorem is the main point of the oral examination question but it relies, of course, heavily on Definition 7.20.

\section{A Priori Analysis of the Situation}

The target knowledge of the situation is the meaning and implications of Definition 7.20, including the fact that at most one number $I$ can satisfy the condition, and the fact that such a number depends only on $\mathbf{V}$ and $\gamma$, despite the presence of the parametrization $\mathbf{r}$ in the definition. As in case 1, a strong didactic obstacle lies in the students' experience of derivatives and integrals as something to be computed according to explicit rule. Specifically, in students' experience, integrals are found by applying computational rules, so that "integrability" appears implicitly as the feasibility of a computation based on rules. The formula in Theorem 7.22 is often used to define curve integrals in calculus courses, and may in fact reinforce this obstacle.

We omit the solution of a); some details will appear in the a posteriori analysis. It is a standard argument based solely on Definition 7.20. For b), the comparison goes as follows: R-exercise 10.3 means that the curve integral is indeed a definite number, depending solely on $\mathbf{V}$ and $\gamma$; but the proof given there is only valid for the more restricted case of $C^{1}$-curves, while the direct argument, to be given in response to question a) of the present exercise, is both simpler and more general. In c), the students should note that $\gamma$ and $\mathbf{V}$ (an oriented curve and a field of vectors for each point on the curve) are indeed "geometric" objects. Thus $\int_{\gamma} \mathbf{V} \cdot d \mathbf{r}$, as a well-defined "function" of $\gamma$ and $\mathbf{V}$, is 
also essentially geometric, unlike a parametrization which is a somewhat arbitrary algebraic description of $\gamma$.

The adidactic potential of the exercise is likely to be enhanced by reminding the students of the common strategy for proving "uniqueness" (the allusion to $I_{1}$ and $I_{2}$ in a)). Concretely, students should be capable of drawing the similar proof of the uniqueness of the Riemann integral, given in the textbook by Eilers et al. (2015), p. 126, while adapting it to the particular properties given in Definition 7.2. This also means that a) has research potential, to engage students in the research like activity of type A5 (mimicking a known proof) and A10 (validating a definition). The adidactic potential in b) and c) depends on the milieus of R-exercise 10.3 and a), and the openness of the questions could be both an obstacle (students will be in doubt on "what do to") and a facility (at least "you can say something"). These questions are otherwise designed in view of their deepening potential, to reflect on the relations and differences between the abstract and general definition 7.20, and the concrete and more constrained algebraic approach provided by Theorem 7.22. It should be noted that the notation $\int_{\gamma} \mathbf{V} \cdot d \mathbf{r}$ could in itself be an obstacle to the points in b) and c), as the symbol " $\mathbf{r}$ " appears in the notation - this might in fact suggest that $\int_{\gamma} \mathbf{V} \cdot d \mathbf{r}$ also depends on a specific parametrization $\mathbf{r}$.

\section{A Posteriori Analysis of the Situation}

Three of the seven focus group students have provided more or less correct solutions to a) (cf. Fig. 1), while they offer at most short and vague remarks to b) and c), such as "The curve integral depends only on the curve and not on the parametrization" (for b)), or "It would be a terrible contradiction if the curve integral depended on $\gamma$ "for c). None of the students note the analogy of a) with the proof of the uniqueness of the ordinary Riemann integral. We can thus affirm that the predicted adidactic and research potentials of a) were realized for these students. On the other hand, the deepening potential associated with b) and c) seems to require a didactic situation of validation and institutionalization, even for these students. The other four students did not produce any written solution prior to class.

The class session has initial phases of collective production, as the teaching assistant asks for ideas:

TA: So we must show that there can be no different $I_{1}$ and $I_{2}$ satisfying this definition. (...) How do we do that?

Student 1: Assume there are two and show the difference is less than $\varepsilon$.

TA: Exact. Quite classical uniqueness thing.

The teaching assistant reminds the class about the analogous proof for the ordinary Riemann integral, and does most of the technical realization of the above idea in oral interaction with other students who, apparently, also solved a) at home: 


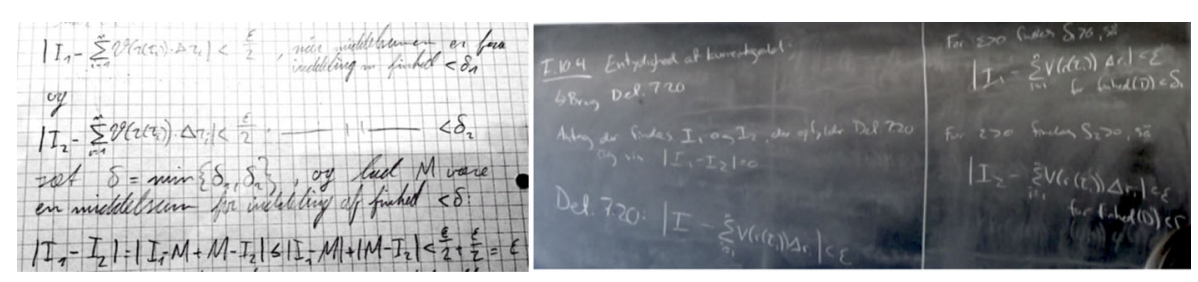

Fig. 1 Student homework (left) and blackboard presentation of question a)

Student 2: Yes but one of the $\delta$ 's must be the smallest. So we just choose $\delta$ to be the smallest of the two.

TA: Mmm, and then what do we do?

Student 2: But then we see that a middle sum with a fineness as our new, a fineness smaller than our new $\delta$ must be, eh, can we chose it so it lies $\varepsilon / 2$ from both middle sums.

TA: Exactly. (...)

We recall that this teaching assistant consistently chose to write do all blackboard writing by herself, presumably to manage time usage and maintain "correctness" of what is written. In more technical moments of work, as with subtle $\varepsilon-\delta$ arguments as above, it becomes more difficult to involve the students, as she does above.

We thus see that the adidactic potential of the first part of the exercise (the more "technical" part) was actually realized for several students. We do not know to what extent this can be ascribed to the relation with old knowledge (further suggested by the notation $I_{1}$ and $I_{2}$ ) and emphasized by the teaching assistant ("Quite classical uniqueness thing"), but at least in the didactic situation it becomes explicit that an old proof technique is used to validate a definition (research like activities A5 and A10).

The deepening potential of questions b) and c) is largely missed. The teaching assistant breaks with the rather permanent principle she has to write solutions to all questions on the blackboard, and simply treats the two questions orally before passing on to the next exercise:

TA: Any questions to this? Everything OK? Perfect. And that's great, 'cause what we have shown here is that if that $I$ exists, that what was I wrote here [points to I], then the $I$ is equal to... [writes $\int \mathbf{V} \cdot d \mathbf{r}$ ] ...to our curve integral. It fits really well what we proved in the exercise before [R-exercise 10.3], that no matter what parametrization you make, there is only one curve integral. That's exactly the same we prove here. There is exactly one every time we have some curve.

The teaching assistant's remark does not go much further than the one line phrases of the students. Clearly, the deepening potential is not realized, as the 
teaching assistant fails to facilitate a discussion of the links between the (general and abstract) definition 7.20 and the (somewhat conditioned) Theorem 7.22 which provides a concrete methods of computation and which is, as already mentioned, the main point of one of the 12 examination questions. While the work with a) has provided a strengthening of knowledge in progress, concerning the technical features of definition 7.20 and its links to older knowledge, the targeted deepening potential concerning the definition and Theorem 7.22 seems to be largely unrealized. One can speculate that the openness of the question is in fact as much of an obstacle as a facility, since both the students and the teaching assistant pass it rather superficially, as if it was trivial. This speculation is strengthened by similar experiences with questions in other exercises that could seem to call for a qualitative judgement, while in fact they pertain to a fundamental point of a theoretical construction in this case, why the theory does not begin with the formula in Theorem 7.22 as a definition, to then just prove the rather simple result of R-exercise 10.3 (as a kind of "uniqueness").

\section{Perspectives}

We have presented two selected cases of exercise design, together with an outline of our analysis of the corresponding didactic situations. On this basis we can now turn to a substantiated discussion of the research questions as well as of more general perspectives of our design methodology.

\section{Theoretical Potentials of Exercises as Design Guideline (RQ1)}

The most fundamental of the four potentials defined in our theoretical framework is the adidactic potential, which is closely related to the milieu that is supposed to resource the students' work with the questions proposed, both in homework and in the didactic situation in class. All exercise construction will, in fact, involve at least implicit assumptions on how those milieus are constituted, and on students' available knowledge (Robert 1998).

Our design methodology, involving a "test solver" (the first author), in fact allowed us to be more explicit in our discussion of these two items, even if the test solver was clearly more advanced in her studies than the prospective students'. As regards the milieu, our discussion of the exercises in Step 3 (after solution of a first version) often led to considerable reformulations that would typically strengthen the "concrete entrance" of the exercise - for instance, it was only in Step 3 that the exercise in case 1 was supplied with the four examples in a). Here, the added adidactic potential is also closely related to the linkage potential, as the consideration of concrete functions could help students establish links between new knowledge (the notion of differentiability in $k$ variables) and old knowledge (partial derivation of functions given in closed form).

In both cases 1 and 2, the exercise begins with a more concrete ("calculus-like") question, incidentally, named a) in both exercises; then follow two more abstract questions, for which the first question and its solution should serve as (part of) the milieu. This way to structure exercises is a natural strategy to pursue adidactic potential, 
in order to build up a richer didactic milieu for a more abstract ("analysis") point. In the exercise case 2, a more recent experience with uniqueness proofs based on the triangle inequality can be "mimicked" to solve the first part; as pointed out in the a priori analysis, the adidactic potential is here associated with the potential of a research like activity (A5). In fact, in all 57 exercises, the adidactic potential is closely linked either to a linkage potential ("old knowledge" should be drawn on) or to a research like activity (often of type A1, A3, or A5).

The research potential was often pursued in relation to a specific point in the textbook (Eilers et al. 2015) - as illustrated by Case 2, which focuses on a definition. Other R-exercises concern a delicate step in a proof. The in-depth study of a specific point in the textbook is of course a quite limited interpretation of "research activity", but given the goals and assessment of the course, it is also a quite important one for our exercise design. In our experience, the differentiated and explicit categories (A1-A10) are very useful guidelines for designing such exercises, focused on "inquiry into a (difficult) mathematical text".

Almost all exercises were designed to have linkage potential or deepening potential (or both), thus enabling the students either to draw on old knowledge from calculus or to elaborate on knowledge in progress (recently or currently studied in the course). We wish to stress the importance to distinguish between these two potentials. They have very different functions in the transition to analysis, as the exercises presented in Case 1 and Case 2 demonstrate: exercises with linkage potential can link (new) theory to (old) practice (Case 1), while exercises with deepening potential aim directly to develop and refine knowledge in progress (Case 2).

To sum up our current answers to RQ1, adidactic potential is an important and necessary notion for all exercise design. With the three other potentials as complements, it was possible to devise a design methodology which resulted in 57 exercises. These exercises provided innovative opportunities for students work with new, theoretical knowledge, while respecting the rather stringent constraints from the institution. We notice that the latter constraints excluded some opportunities such as significant use of computer algebra systems (Gyöngyösi et al. 2011) or exercises delving into important links with other disciplines such as mechanics. We return to other effects of these constraints later in this section.

\section{Realizations of Potentials and the Possibility of Redesign (RQ2)}

The two cases illustrate rather well that the potentials could not be observed from the classroom situations alone, given the choice of the teaching assistant to do all the blackboard presentation, with occasional prompts for input from the students. But students' main work with exercises was to be completed before then, and we have other data to shed some light on that as well.

In case 1, we saw that the adidactic potential was actually too weak for most students; in homework, no students succeeded completely with the four examples in question a), let alone the two follow-up questions. On the other hand, most of them were able to at least make some first experiments with the four functions and in the classroom situation, even those who did not solve a) beforehand were able to draw on the milieu of the solutions to a) - given in class - to contribute to the solution of b) and 
c). To redesign the first part of the task one should focus on creating a richer milieu for the students, resulting in better adidactic potential. This could be done by inserting a new question before a): use an appropriate tool to draw the graphs of the functions and use it to make a guess on whether they are differentiable. This, of course, amounts to an adaptation of the exercise to the observed lack of spontaneous engagement of the students in a heuristic investigation (A6), in order to strengthen the research potential of the exercise.

On the other hand, case 2 shows a somewhat opposite outcome: the adidactic potential of a) seems to have sufficed for a good number of students to develop a reasonable answer to that question, but the students do not respond well to b) and c) as they are formulated, and even the teaching assistant does not really institutionalize the main points. The teaching assistant could of course have managed the didactic situation differently, to draw more on the work actually done by the students, as a milieu for these crucial theoretical questions. To achieve a higher adidactic potential of the questions, one could again consider reformulations of them that were less open and more explicit in their demands related to the deepening potential, like "Here is an alternative definition of the curve integral that would save us most of the work... what could be the reasons the authors did not choose it? What do they achieve by not doing it?"

Both cases illustrate a tendency (in redesign or even in actual teaching) of reduction of tasks which is both well known, difficult to avoid and certainly important to control: to enhance or even enforce the chances of students achieving a target knowledge in a situation, one is tempted to reduce the task left to the student, thereby in fact reducing the adidactic potential. In ordinary teaching, if the students fail to adapt to the proposed milieu in a didactic situation, we naturally modify the milieu, while trying to preserve some adidactic potential. For an exercise, the appropriate resistance of the milieu has, in principle, to be gauged beforehand. In the setting of a course, however, one could also consider to split the exercise in two, with only some parts left as homework. This would defy the constraints at hand (only change exercises), as it would require a more controlled design of the didactic situation in class to optimize the adidactic potential there. In our context, the way class sessions were taught was left entirely to the teaching assistants.

Moving now to more global observations than what can be based on the two cases alone, we would like to mention the following facts (which, clearly, are more loosely connected to RQ2):

- Among the seven focus group students, whose preparation was followed throughout the course, it was on average 4.5 who brought notes for the exercises of a given section, mostly partial solutions, as the ones considered in the two cases; two of the seven students only prepared notes ahead of one out of 11 sessions, so it was almost always the same students who met with written preparations.

- From the anonymous, written course evaluation, answered by 82 out of 295 students, it appears that less than half of the students worked on the exercises before the class sessions, that they tend to find the exercises difficult, and that few were able to solve all of them (see Table 1 for exact figures).

- At the oral exams, of which 32 were observed by the first author, it appeared that Rexercises were mostly referred to by high performing students, and also that some of the exercises (linked to specific questions) were more often referred to than others; in particular, explicit use of exercises with research potentials linked to A3 and A5 
Table 1 Students' evaluation of R-exercises (number of students, with a total of 82 respondents)

\begin{tabular}{llllll}
\hline Please mark the extent to which you agree with the following: & 1 & 2 & 3 & 4 & 5 \\
\hline I normally try to solve the R-exercises before the class sessions & 8 & 15 & 25 & 28 & 6 \\
The R-exercises are normally easy to get started with & 10 & 20 & 25 & 26 & 1 \\
I have been able to solve almost all R-exercises before class & 17 & 33 & 19 & 11 & 2 \\
The R-exercises appear to be relevant for the oral exam & 0 & 4 & 15 & 41 & 22 \\
\hline
\end{tabular}

$1=$ Disagree completely $2=$ Disagree $3=$ Neither agree or disagree $4=$ Agree $5=$ Agree completely

appear quite often, which is natural given the focus on textbook proofs at the exam. This is also confirmed by focus group interviews with students after the exam.

- Also at the oral exam, low performing students often got stuck with formulating and explaining basic definitions; exercises focusing on A10 could, for this and other reasons, be interesting to prioritize in future designs.

\section{Reasons for Discrepancies Between a Priori and a Posteriori Analysis (RQ3)}

There is nothing surprising or problematic in the general observation that expected potentials for student work with the R-exercises were only partially realized. As we have seen, even adidactic potential need not be realized in students' private work in order for this and other potentials to be, at least to some extent, realized in class sessions. As we have already mentioned, redesign with respect to clearer boundaries between work to be done before class, and complements to be achieved in the didactic situation of the class, might be helpful if institutionally feasible. At the same time, redesign for higher adidactic potential of exercises is an important endeavor that should also improve the realization of the other, more specific potentials - which all depend on the first one.

But no matter how well adapted exercises are to students' prerequisites and the learning goals of the institution, the actual engagement of students in the work is indeed a necessary condition for the realization of any potential. It is an eye-catching tendency, visible in Table 1, and also from the detailed presentation of the two cases, that only often less than - half of the students seem to find the time to even try to solve the exercises by themselves. This, naturally, cannot be explained by the exercises alone although Table 1 also indicates that roughly half of the students found the exercises "difficult" to get started with, and thus could suggest an insufficient adidactic potential of many of them (this is also confirmed by other sources).

Besides these two major general reasons - students' insufficient investment of time in homework, and insufficient adidactic potential of (some) exercises - we should also point to a third major reason for unrealized theoretical potentials, which is well illustrated by case 2: the class session themselves often take the form of "lecture style" presentation of exercise solution by the teaching assistant, a phenomenon which is not limited to this class and teaching assistants who were observed systematically throughout the course. Naturally, the choice of the institution to confer this part of the course to experienced students, rather than faculty, is common in other similar contexts, and has several rationales (financial, 
experienced students as role models, academic profit for them to teach younger students etc.). Moreover, the time constraints - visible throughout our observations of the specific class - would not disappear with more mature class teachers; indeed, the adoption of alternative priorities and modes of instruction (such as not presenting all details of solutions on the blackboard) would depend on deliberate, institutionally backed decisions, regardless of the class teachers. And as even some of the teaching assistants claim to have "never solved an exercise before class" when they themselves took the course, it seems clear that the problem of non-engagement of many students is more linked to institutional norms than to the character of the exercises or even the capacities of the teaching assistants. Still, it seems obvious to us that implementing major changes in the norms and teaching modes of the institution, particularly when it comes to exercise sessions, would require a deliberate effort by leading faculty members and the institution management.

\section{Conclusions}

The TDS-based notions of adidactic potential, linkage potential, deepening potential and research potential were introduced as tools to design exercises for a first course in analysis, within a specific context that reflect more common transition phenomena at the interchange between calculus and analysis. In particular, we have presented a detailed framework for research potential, formulated as concrete activities which exercises could be constructed to engage students in, and this seems to us to furnish firm and helpful guidelines for the framing of student work in such a course. The framework of the four potentials, in particular the notion of research potential, is a main contribution of the paper which can be invested (and further developed) in new studies in our or similar contexts. In this paper, we have not been able to present and analyse more than two different cases, but the two cases illustrate the use of all four potentials, including a wide range of the research like activities.

In this paper, the notions were deployed to analyze the outcomes within a very specific context, where students' homework and observation of class sessions are the most important sources of information about the extent to which the potentials of an exercise were realized. We wish to conclude by some perspectives arising from our observations in this context. It should be stressed that there is no surprise in finding unrealized potentials. As in any design process, such findings could serve as a basis for further development of the specific exercise design. In particular, in Case 1 (question a)) we have noticed the need to enrich the milieu with more explicit indications on the use of graphical representations, and the delicacy of devolving and managing the more abstract questions (b), c)) considered in Case 2.

The limits of what can be controlled by exercise design are also apparent in the two cases and in the more general observations made in the previous section. While an institutional analysis of the constraints encountered by this project is out of the scope of the present paper, we wish to point out a few which are likely to find counterparts in other institutions, and which may in fact be changed at a local level.

It appears from the cases presented, as from other observations we made, that the didactic situations in the classrooms did not fully exploit the potential of students' work both before and during the classes. This can to a large extent be ascribed to the customs and capacities of the teaching assistants who direct those classes. They are successful 
"older" students who took the course some years ago, and simply repeat the pattern of "teacher presentation of solutions" they met there. This may in fact be found to reduce the adidactic potential of exercises as germs of situations in real time teaching. It is an obvious and manageable idea to introduce them to alternative modes of classroom management, and also to at least some of the rationale behind the R-exercises (as presented here).

Similarly, students' expectations as to what work is important and worthwhile to prioritize within a course may be surprisingly immune to explicit statements about the alignment of exercises to course requirements (here, the oral exam). Some of the solution may lie in the redesign of exercises, in particular to increase the adidactic potential further. But as the beliefs of students are often influenced by "older" students, it may take two or three editions of a course before students adapt to changes in the contents of the teaching and examination, even if they are carefully aligned.

The institutional constraints on students' and teachers' work are certainly important factors to explain the observed distances between realized and theoretical potentials. In this paper we have focused on the more local ones and, admittedly, taking them more or less as boundary conditions which we were not able to change. Clearly constraints such as time frames, examination rules and syllabi cannot be ignored and it may sometimes be both necessary and possible to change them. At the same time, we are convinced that "local" interventions based on task design (exercises together with an explicit a priori analysis), using the categories and methods presented here, can contribute the solving real problems in a wide range of mathematics programmes that are struggling with similar transition problems between calculus and analysis.

\section{References}

Brousseau, G. (1997). Theory of didactical situations in mathematics: didactique des mathématiques, 19701990 (Mathematics education library, Vol. 19). Dordrecht; Boston: Kluwer Academic Publishers.

Burton, L. (2004). Mathematicians as enquirers: Learning about learning mathematics. Netherlands: Springer.

Eilers, S., Hansen, E., \& Madsen, T. G. (2015). Indledende matematisk analyse. Copenhagen: University of Copenhagen.

González-Martín, A. S., Bloch, I., Durand-Guerrier, V., \& Maschietto, M. (2014). Didactic situations and didactical engineering in university mathematics: cases from the study of calculus and proof. Research in Mathematics Education, 16(2), 117-134. doi:10.1080/14794802.2014.918347.

Gravesen, K. (2015). Forskningslignende situationer i et første teoretisk kursus i reel analyse (Research like situations in a first theoretical course on real analysis). MSc thesis in mathematics, University of Copenhagen.

Gyöngyösi, E., Solovej, J. P., Winsløw, C. (2011). Using CAS based work to ease the transition from calculus to real analysis. In Pytlak, M., Rowland, T., Swoboda, E. (Eds.), Proceedings of the seventh congress of the European society for research in mathematics education European Society for Research in Mathematics Education, 2002-2011.

Hersant, M., \& Perrin-Glorian, M.-J. (2005). Characterization of an ordinary teaching practice with the help of the theory of didactic situations. Educational Studies in Mathematics, 59(1-3), 113-151. doi:10.1007 /s10649-005-2183-z.

Joubert, M. (2013). Using computers in classroom mathematical tasks: revisiting theory to develop recommendations for the design of tasks. In C. Margolinas (Ed.), Task design in mathematics education. Proceedings of ICMI study 22 (Vol. 1, pp. 71-79). Oxford: ICMI. 
Madsen, L. M., \& Winsløw, C. (2009). Relations between teaching and research in physical geography and mathematics at research-intensive universities. International Journal of Science and Mathematics Education, 7(4), 741-763. doi:10.1007/s10763-008-9134-y.

Misfeldt, M. (2006). Mathematical Writing. PhD dissertation, Aarhus University, Copenhagen.

Ouvrier-Buffet, C. (2011). A mathematical experience involving defining processes: in-action definitions and zero-definitions. Educational Studies in Mathematics, 76(2), 165-182.

Robert, A. (1998). Outils d'analyse des contenus mathématiques à enseigner au lycée et à l'université. Recherches en Didactique des Mathématiques, 18(2), 139-190.

Winsløw, C. (2008). Transformer la théorie en tâches: la transition du concret à l'abstrait en analyse réelle. In A. Rouchier \& I. Bloch (Eds.), Actes de la XIIIème école d'été en didactique des mathématiques. La Pensée Sauvage: Grenoble.

Winsløw, C., Barquero, B., De Vleeschouwer, M., \& Hardy, N. (2014). An institutional approach to university mathematics education: from dual vector spaces to questioning the world. Research in Mathematics Education, 16(2), 95-111. doi:10.1080/14794802.2014.918345. 